\title{
Oscilaciones cerebrales: papel fisiopatológico y terapéutico en algunas enfermedades neurológicas y psiquiátricas
}

\section{Brain oscillations: pathophysiological and therapeutic role in some neurological and psychiatric diseases}

\author{
J. Artieda, M. Alegre, M. Valencia, E. Urrestarazu, M. Pérez-Alcázar, M.J. Nicolás, \\ J. López Azcárate, J. Iriarte
}

\section{RESUMEN}

Se usa el término "oscilación o actividad oscilatoria" para referirse a las fluctuaciones rítmicas de los potenciales postsinápticos de un grupo neuronal (potenciales de campo local) o de una región cortical (EEG electrocorticografía) y también al patrón de descarga rítmico de los potenciales de acción de una neurona o un grupo neuronal. La actividad oscilatoria posibilita la sincronización entre grupos neuronales de la misma área cortical o de áreas distantes entre sí que intervienen en una acción motora, tarea cognitiva o perceptiva. Con frecuencia es motivo de confusión asociar la presencia de actividad oscilatoria con fenómenos de sincronización, ya que ambos fenómenos aunque relacionados no son equivalentes. En patologías neurológicas o psiquiátricas tan distintas como la enfermedad de Parkinson u otros movimientos anormales, la epilepsia o la esquizofrenia se han descrito anomalías de la actividad oscilatoria de distintas estructuras cerebrales o de su sincronización que podrían jugar un papel relevante en su fisiopatología. En esta revisión se discuten estos aspectos haciendo hincapié en su importancia por ser un mecanismo básico del funcionamiento cerebral y un nuevo mecanismo fisiopatólogico de la sintomatología de algunas enfermedades cerebrales.

Palabras clave. Oscilaciones. Ganglios basales. Enfermedad de Parkinson. Epilepsia. Esquizofrenia.

\begin{abstract}
The terms "oscillations" or "oscillatory activity" are frequently used not only to define the rhythmic fluctuations of the postsynaptic potentials of a neuronal group (local field potentials) or a cortical region (EEG, MEG), but also to indicate the rhythmic discharge pattern of action potentials from a neuron or a small group of neurons. Oscillatory activity makes possible the synchronization of different neuronal groups from nearby or distant cortical regions that participate in the same motor, sensory or cognitive task. The presence of oscillatory activity is usually associated to the existence of synchronization, but both phenomena are not necessarily always equivalent. Abnormalities of oscillatory activities or synchronization within or between different brain structures have been described in several neurological and psychiatric diseases; these abnormalities might play a relevant pathophysiological role in Parkinson's disease (and other movement disorders), schizophrenia or epilepsy. This review discusses all these aspects, with emphasis on their potential role both as a basic mechanism in brain function and as a pathophysiological substrate for some of the symptoms and signs observed in several diseases.
\end{abstract}

Key words. Oscillations. Basal ganglia. Parkinson`s disease. Epilepsy. Schizophrenia.
Servicio de Neurofisiología Clínica.

Área de Neurociencias. CIMA y Clínica

Universidad de Navarra. Pamplona.

\author{
Correspondencia \\ Julio Artieda \\ Servicio de Neurofisiología Clínica \\ Clínica Universidad de Navarra \\ Apdo. 4209 \\ 31008 Pamplona. España \\ Tfno. +34948255400 Ext 4550 \\ Fax +34948296500 \\ E-mail: jartieda@unav.es
}




\section{INTRODUCCIÓN}

La existencia de actividad oscilatoria cortical es un hecho bien conocido desde los primeros registros electroencefalográficos. Los ejemplos más llamativos en el hombre son: el ritmo alfa occipital durante la vigilia con ojos cerrados, el ritmo mu rolándico o las distintas actividades del sueño -husos de sueño, theta y delta-.
Se usa el término "oscilación" o "actividad oscilatoria" para referirse a las fluctuaciones rítmicas de los potenciales postsinápticos de un grupo neuronal (potenciales de campo local) o de una región cortical (EEG, electrocorticografía), pero también al patrón de descarga rítmico de los potenciales de acción de una neurona o un grupo neuronal (Fig. 1).

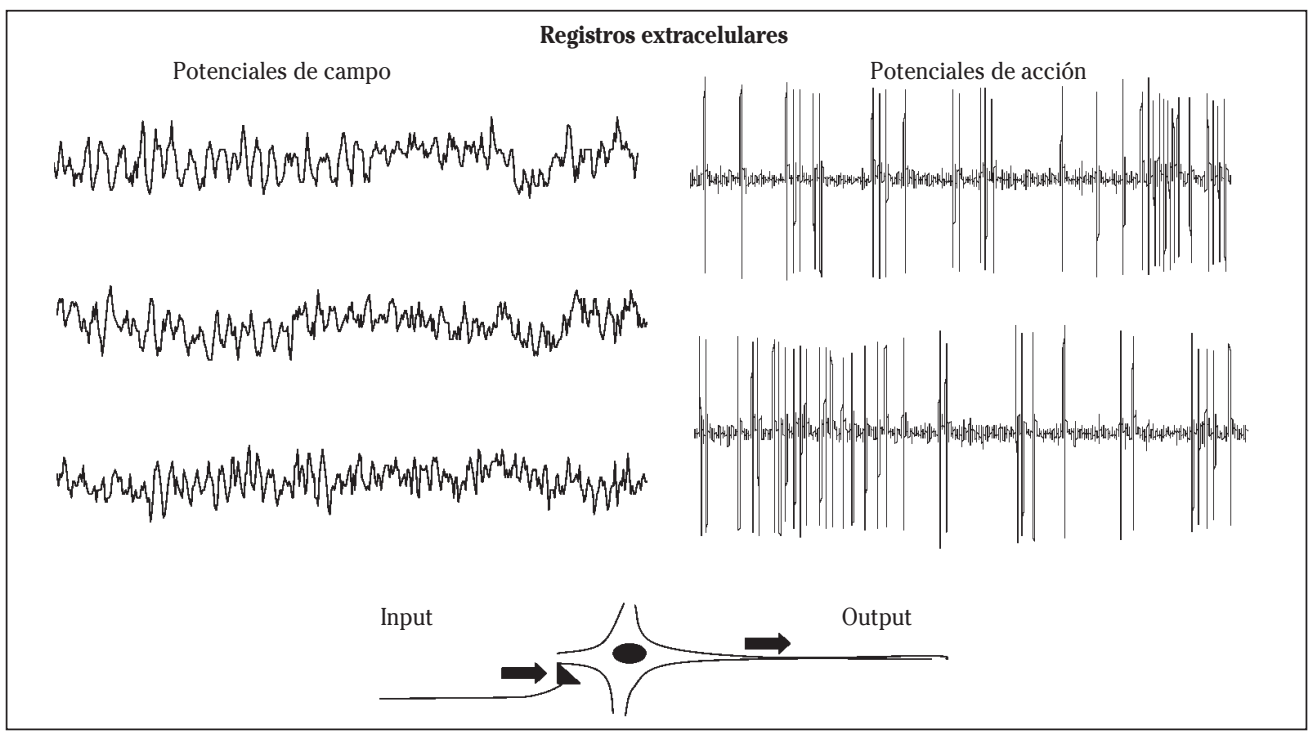

Figura 1. Diferencias entre el comportamiento oscilatorio de los potenciales de campo local (izquierda) y los potenciales de acción (derecha). En ambos casos se utiliza el término oscilación para expresar fenómenos fisológicamente diferentes. Las oscilaciones de los potenciales de campo expresan la aferencia a un núcleo y son fruto de la suma de potenciales postsinapticos mientras que los potenciales de acción expresan la eferencia.

Ambos tipos de oscilaciones expresan como muestra la figura 1 fenómenos fisiológicos diferentes. Las oscilaciones registradas en el EEG, electrocorticografía o potenciales de campo local reflejan el resultado de la suma de los potenciales postsinápticos generados en la región próxima al electrodo de registro (es decir, la entrada de información o aferencia a dicha zona), mientras que los potenciales de acción son la respuesta (eferencia) de las neuronas situadas en torno a la punta activa del electrodo.

Existe un acuerdo general en que la actividad oscilatoria constituye un mecanis- mo básico del funcionamiento cerebral. La actividad oscilatoria aparece a diferentes niveles cerebrales, participando en procesos perceptivos, cognitivos y motores. La actividad oscilatoria y sincronización son fenómenos distintos, aunque asociados. La actividad oscilatoria facilita la sincronización de la descarga de potenciales de acción de grupos neuronales o áreas corticales, cercanas o distantes.

A continuación se discuten y revisan diferentes aspectos relacionados con la fisiología de la actividad oscilatoria cortical, las distintas metodologías que existen para 
su estudio y su papel en la fisiopatología de algunos trastornos neurológicos y psiquiátricos.

\section{OSCILACIONES Y SINCRONIZACIÓN. EL PROBLEMA DEL «BINDING»}

La primera descripción de actividades rápidas espontáneas en la banda gama re- monta a los años 40 donde se estudiaron las oscilaciones registradas en el bulbo olfativo de algunos animales. Pero fue a principios de los años 90, con la descripción la actividad oscilatoria y su sincronización realizada por el grupo de Singer ${ }^{1}$, cuando estos fenómenos comenzaron a considerarse un mecanismo básico del funcionamiento cerebral y el interés en la comunidad científica se disparó (Fig. 2).

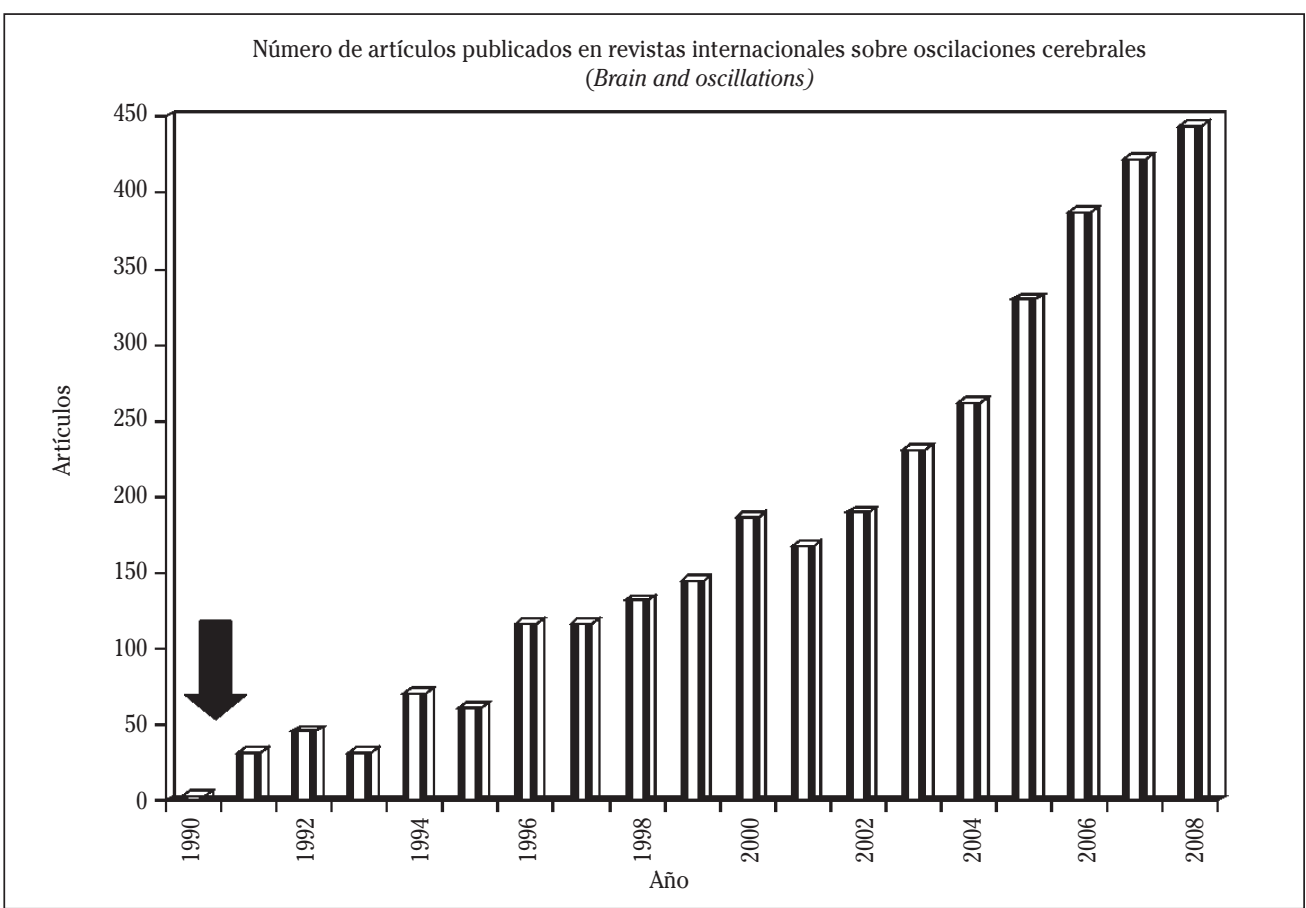

Figura 2. Evolución en los últimos años del número de publicaciones referenciadas que tratan sobre oscilaciones cerebrales. Se puede observar el interés creciente en el tema.

La visión de un objeto, la audición de una palabra o la percepción de un elemento complejo conlleva la activación de distintos grupos neuronales, columnas o incluso áreas corticales muy distantes entre sí. Cada una de ellas codifica una característica elemental del objeto. La combinación de cada una de esas características aisladas en una única representación del objeto constituye un proceso complejo, habitualmente conocido como "binding" (unión, integración) ${ }^{2}$.

Se han elaborado diversas teorías sobre el "binding" perceptivo. El modelo más simple es el patrón jerárquico piramidal: la suma de descargas neuronales asociadas a las características más simples genera la activación de neuronas progresivamente más específicas hasta llegar finalmente a 
una única neurona asociada al objeto en cuestión ${ }^{3}$. Esta teoría presenta dos importantes limitaciones: la explosión combinatoria (no existen en el cerebro suficiente número de neuronas para reconocer cada uno de los posibles objetos que podemos encontrar en el mundo que nos rodea) y la necesidad de disponer de un gran número de neuronas de "reserva" esperando a aprender a reconocer los objetos nuevos que descubrimos cada día. Como alternativa, Hebb propuso un modelo en el que la percepción global de un objeto vendría dada por el aumento en la intensidad de descarga de cada una de las neuronas implicadas en el reconocimiento de sus características ${ }^{4}$. Esa combinación de descargas daría lugar a un patrón distinto para cada uno de los objetos posibles, evitando el problema de la explosión combinatoria (número casi infinito de posibles combinaciones de descarga de distintas neuronas). Sin embargo, este modelo presenta un nuevo problema, la denominada "catástrofe de superposición": ante la presencia de varios objetos diferentes, aumentaría la descarga a la vez de todas las neuronas o grupos neuronales implicados en el reconocimiento de las distintas características de cada uno de ellos, resultando imposible distinguir cuáles de ellas corresponderían a cada objeto. Von der Malsburg propuso el modelo más aceptado actualmente ${ }^{5,6}$. La «integración» de las distintas características del objeto no se consigue mediante un aumento en la frecuencia de descarga de las neuronas correspondientes, sino mediante la sincronización de dichas descargas. De esta forma, se permite la percepción simultánea de diversos objetos, ya que pueden sincronizarse a distintas frecuencias o con distinta fase muchos grupos diferentes.

La sincronización se ve facilitada en mayor medida cuando las neuronas implicadas en la misma presentan actividad oscilatoria de manera espontánea. Estas oscilaciones (y la sincronización correspondiente) ocurren en un rango amplio de frecuencias. Se han descrito oscilaciones en relación con fenómenos de integración en múltiples tareas perceptivas, cognitivas o motoras. El ejemplo más prototípico lo conforma la actividad en la banda gamma durante los periodos de actividad en las zonas correspondientes de la corteza cerebral, junto con la sincronización observada entre distintos grupos neuronales en dicho rango de frecuencias.

Así pues, las oscilaciones no aportan información en sí mismas. Más bien, actúan como el substrato que permite la integración de la información que llevan las distintas neuronas que se sincronizan, funcionando como una especie de "semafóro" que controla la descarga de las neuronas excitadoras.

\section{ORIGEN DE LA ACTIVIDAD OSCILATORIA. REDES NEURONALES Y SISTEMAS COMPLEJOS}

Existen múltiples mecanismos capaces de generar actividad neuronal oscilatoria. El más simple consiste en la descarga rítmica espontánea de una o un conjunto de neuronas, como ocurre en las células del sistema de conducción cardiaco. Sin embargo, la actividad oscilatoria puede ser generada más fácilmente mediante circuitos neuronales. En estas redes juegan un papel fundamental las interneuronas inhibitorias ${ }^{7}$. Existen modelos teóricos de redes neurales oscilatorias e incluso evidencia experimental que apoya su existencia. Las oscilaciones cerebrales y su frecuencia vienen condicionadas por la estructura del sistema nervioso y más concretamente por las características morfólogicas de la misma red. El número de sinapsis, de interneuronas, la longitud de los axones y la velocidad de conducción de éstos condicionan la dinámica de la red neuronal y por tanto las oscilaciones que emergen. El cerebro de la rata presenta un menor número de sinapsis, un menor número de interneuronas y distancias menores entre los distintos elementos de la red, debiendo generar obligatoriamente oscilaciones a frecuencias diferentes a las del cerebro del hombre ${ }^{8}$. En general, se acepta que las oscilaciones de alta frecuencia se originan en pequeñas poblaciones neurona- 
les, mientras que las oscilaciones de baja frecuencia comprenden poblaciones más amplias $^{9,10}$. Estas redes neuronales oscilatorias pueden ser puramente corticales, pero existen evidencias especialmente basadas en el estudio de la actividad oscilatoria de sueño, que sugiere que el tálamo juega también un papel importante en el mantenimiento de estas oscilaciones rítmicas. En otras estructuras subcorticales, como la corteza cerebelosa o los ganglios basales también se han descrito oscilaciones a distintas frecuencias.

La variabilidad, recurrencia y diversidad observada en los patrones de oscilación, unido al hecho de que se encuentren a diferentes escalas o niveles del sistema nervioso sugiere que, por un lado, los mecanismos que originan la actividad oscilatoria cerebral son complejos y que además tienen su origen en las intricadas relaciones establecidas entre los diferentes elementos que lo conforman.

La observación de un sistema cuyo alto grado de complejidad es debido a la intrincada red de relaciones establecida entre los elementos que lo forman no es exclusivo del cerebro; en la naturaleza existen multitud de ejemplos en los que los fenómenos (inundaciones, revueltas sociales, terremotos o maremotos) observados dentro del sistema (clima, redes sociales, corteza terrestre o el mar) son el resultado de las relaciones establecidas entre sus elementos (masas de gas, personas, placas tectónicas o corrientes marinas). Las dinámicas observadas en estos sistemas tienen su origen en el elevado número de grados de libertad que poseen. Por un lado, están constituidos por infinidad de elementos interaccionantes; pero además, estas relaciones frecuentemente presentan un fuerte carácter no lineal, lo que da a lugar a fenómenos tales como la presencia de correlaciones temporales de largo alcance, fenómenos de criticalidad, existencia de memoria en el sistema, auto-organización de sus elementos, u otras propiedades emergentes.

Cabe resaltar que a diferencia de los denominados sistemas "complejos", existe otro tipo de sistemas, que podemos denominar como "complicados" y que resultan de la simple adición de sus partes. Este tipo de sistemas no presentan la capacidad de evolucionar u organizarse si no es mediante la actuación de intervenciones externas. De forma aproximada, podemos entender que un sistema complicado es un sistema que ha sido "diseñado". Ejemplos de ello lo constituyen un televisor o un vehículo. Si bien ambos están conformados por multitud de piezas, requieren de la intervención de un diseñador para modificar su comportamiento y difícilmente pueden realizar otras tareas diferentes a aquellas para las que fueron concebidos.

Contrariamente, en los sistemas "complejos" puede decirse que "el todo es mucho más que la suma de sus partes"; los comportamientos observados surgen como consecuencia de interacciones entre los elementos del sistema, y en ningún caso pueden inferirse con la superposición de los elementos aislados ${ }^{11}$. Básicamente, este tipo de sistemas posee propiedades que emergen de la dinámica de sus partes de las relaciones establecidas entre las mismas. Por ejemplo, cuando observamos el comportamiento de un hormiguero, el comportamiento individual de cada hormiga resulta muy elemental, pero el comportamiento conjunto del hormiguero denota una complejidad enorme, ya que muestra características que evidencian por ejemplo, la existencia de memoria dentro de la comunidad a lo largo de múltiples generaciones, si bien, la vida de una hormiga se reduce a unos pocos días ${ }^{12}$.

Todas estas propiedades, denominadas emergentes, surgen como fruto de la propia auto-organización del sistema. Como hemos visto, un cambio evolutivo en el coche o en el aparato de televisión está sujeto a la intervención de un supervisor, mientras que en sistemas como el hormiguero no es posible detectar un "director de orquesta", sino que los elementos que conforman el sistema se autoorganizan de forma que dan lugar a comportamientos comunitarios que posibilitan la evolución del sistema ${ }^{11,13}$. 
Otra característica que frecuentemente presentan los sistemas complejos es la posibilidad que tienen para trabajar en un punto próximo a la criticalidad. Si bien el córtex cerebral constituye un sistema eminentemente excitatorio, también dispone de mecanismos inhibitorios que evitan una difusión indiscriminada de la actividad neuronal, a la vez que permiten transmitir la información entre cualquier par de puntos del cerebro. Por lo tanto, ha de existir un equilibrio que permita que una neurona excite a un número suficientemente elevado de neuronas de forma que logre transmitir la información que codifica, pero a la vez, ese número sea lo suficientemente pequeño como para que no desencadene una cascada de activaciones que cubra todo el cerebro. En cualquiera de los dos extremos el comportamiento del sistema no resultaría crítico; si el número de neuronas alcanzadas resultara excesivamente elevado, el sistema estaría trabajando en un régimen super-crítico y colapsaría el cerebro, mientras que si el número de activaciones fuera insuficiente, el sistema estaría inmerso en un régimen sub-crítico con lo que no sería capaz de transmitir la información de forma eficiente. El régimen de funcionamiento óptimo sería por lo tanto el crítico, aquel en el que ambos regímenes (opuestos) están balanceados ${ }^{11-14}$.

La concepción de sistema críticamente auto-organizado fue identificada por Per Bak y col en un artículo inaugural publicado en Physical Review Letters en $1987^{13}$. Dicho trabajo considera algunos de los mecanismos por los cuales la complejidad en los sistemas es un fenómeno tan común en la naturaleza. Sus conceptos se han aplicado de manera entusiasta a distintas ciencias. El concepto de sistema críticamente auto-organizado se ha utilizado para explicar tanto la impredecibilidad de los terremotos, los incendios o la extinción de especies. Las redes neuronales también constituyen un claro ejemplo de sistema críticamente auto-organizado; presentan un alto grado de complejidad, fruto del elevado número de elementos (no-lineales) que lo componen y de la forma en que éstos se interrelacionan.

Estudios iniciales han demostrado que la actividad electroencefalográfica presenta correlaciones a largo plazo ${ }^{14}$, un fenómeno característico de sistemas complejos con este tipo de dinámicas. Igualmente, modelos matemáticos que simulan en el ordenador redes neuronales (como puede ser la corteza cerebral), tienen comportamientos complejos. Simulaciones de redes de neuronas presentan actividad oscilatoria y formas de propagación de la actividad, semejante a las que se han observado en la corteza cerebral de la rata, apreciándose ondas en espirales, que denotan la transmisión de la información dentro de un tejido con heterogeneidades que podrían cambiar en el tiempo.

Disminuciones en la inhibición (como ocurre por ejemplo en la enfermedad de Parkinson, dentro de la corteza cerebral y a otros niveles) dan lugar a cambios en las frecuencias y características de la oscilaciones. De esta forma, la aparición o modificación de la actividad oscilatoria podría considerarse como un indicador de las propiedades emergentes que poseería el cerebro, modelado como un sistema complejo bajo el axioma de criticalidad autoorganizada; las oscilaciones deberían mantenerse dentro de un umbral crítico, que permita una respuesta rápida y flexible. Según esta hipótesis, un sistema rígido con oscilaciones invariables podría resultar tan patológico como un sistema caótico con oscilaciones incontrolables.

\section{ALGUNOS MÉTODOS DE ESTUDIO DE LAS OSCILACIONES Y LA SINCRONIZACIÓN}

Existen posibles aproximaciones muy diversas al estudio de la actividad oscilatoria y de la sincronización cortical. En primer lugar, hay que distinguir los métodos que se aplican sobre señales de potenciales de acción (neurona única o multiunidades) frente a aquéllos que es- 
tán diseñados para estudiar potenciales de campo (incluyendo el EEG, la ECoG y la MEG). En segundo lugar, existen métodos que estudian las oscilaciones a partir de una única señal (univariados) y métodos para estudiar la sincronización ente dos (o más) señales con orígenes diferentes (bivariados/multivariados). Por último, deben distinguirse métodos estáticos (que miden las oscilaciones o la sincronización en el conjunto de un periodo donde la señal es estable) y dinámicos, que miden el cambio en las oscilaciones o la sincronización a lo largo del tiempo. Algunos de estos aspectos se expondrán por separado.

\section{Análisis de potenciales de acción y de potenciales de campo}

Los potenciales de acción son un fenómeno todo o nada (siempre tienen las mismas características). Por tanto, pueden representarse en forma de una serie de eventos a lo largo del tiempo. Si disponemos de un registro de una única neurona, basta con establecer un "nivel» a partir del cual consideramos que ha habido un potencial de acción y marcamos un evento cada vez que se cruza ese umbral. En un registro que incluya varios potenciales de acción, es necesario aplicar sistemas automatizados de reconocimiento de patrones para diferenciarlos. Una forma sencilla de apreciar la ritmicidad de las descargas consiste en elaborar un histograma que represente la probabilidad de un evento a lo largo del tiempo en relación con la aparición de un evento anterior (autocorrelograma). Una actividad rítmica dará lugar a una oscilación reconocible en ese histograma. Lo mismo puede hacerse para comprobar la sincronización entre la descarga de dos neuronas: se representa la probabilidad de descarga de una de ellas en función de la descarga de la otra a lo largo de un periodo de tiempo determinado, habitualmente corto (correlación cruzada). Para realizar análisis más detallados, se pueden con- vertir los eventos (señal discreta) en una señal continua representando el intervalo de tiempo transcurrido entre cada uno de ellos (o su inverso, la frecuencia instantánea) o la frecuencia media en un intervalo de tiempo determinado. A esta nueva señal continua (con una "frecuencia de muestreo" determinada por el intervalo de tiempo elegido para calcular la frecuencia media) se le pueden aplicar las técnicas de análisis de señales continuas que se describen a continuación, utilizadas habitualmente con los potenciales de campo. De esta forma pueden encontrarse oscilaciones más lentas que a priori pasarían inadvertidas ${ }^{15}$.

\section{Herramientas de estudio de las oscilaciones en una señal continua}

La herramienta más básica en el estudio de las oscilaciones de una señal neurofisiológica es la transformada rápida de Fourier, que proporciona el espectro de potencia de la señal estudiada (Fig. 3). Esta herramienta da una medida estática, es decir, mide la contribución de cada frecuencia a la señal sin tener en cuenta el factor temporal. Si lo que se desea es medir los cambios en las oscilaciones a diferentes frecuencias a lo largo del tiempo (medidas dinámicas), se puede recurrir a técnicas basadas en transformadas tiempo-frecuencia ${ }^{16}$. Estas técnicas proporcionan una representación (habitualmente representada como una figura en color o 3-D) que muestra la evolución de la energía correspondiente a cada frecuencia a lo largo del tiempo. Existen diversos tipos de transformaciones tiempo-frecuencia, siendo una de las más utilizadas la basada en "wavelets»"

Existen además otro conjunto de herramientas que permiten estudiar no ya el comportamiento de una frecuencia concreta en una señal, sino las interacciones entre distintas frecuencias dentro de esa misma señal. Un análisis más detallado de las mismas queda fuera del objetivo de este trabajo. 


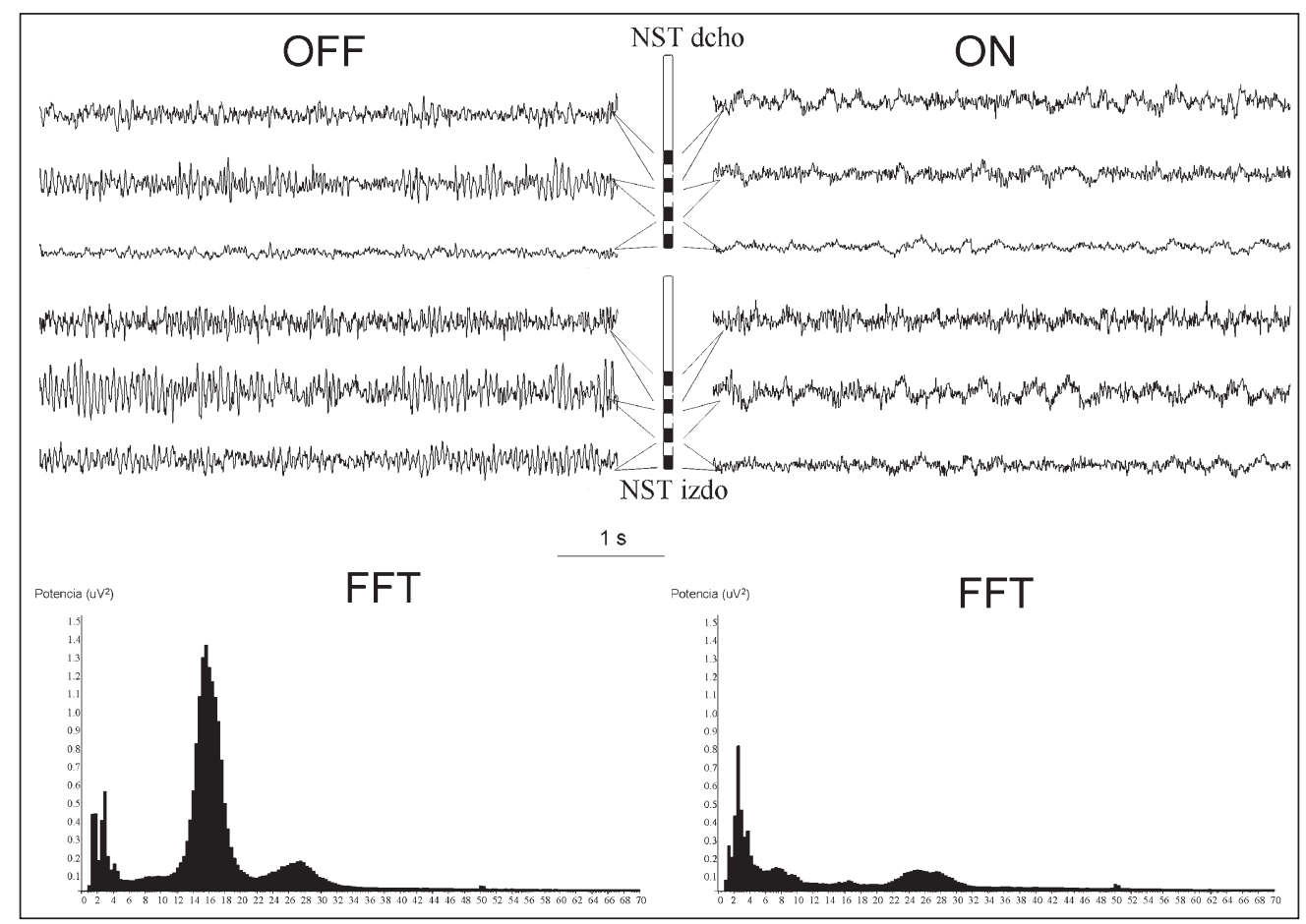

Figura 3. Actividad oscilatoria en el núcleo subtalámico (potenciales de campo local) en paciente con enfermedad de Parkinson. El registro se ha realizado a través de los electrodos de estimulación profunda. En la parte de arriba se muestra el registro y abajo el espectro de potencia obtenido mediante aplicación de una trasformada rápida de Fourier. Se puede observar un pico de actividad beta en "off» que desaparece en situación "on".

\section{Medición de la sincronización entre dos señales continuas}

La medida más comúnmente utilizada para evaluar la existencia de «sincronización" entre dos señales registradas es la coherencia. La coherencia mide hasta qué punto existe una relación lineal (en términos de amplitud y fase) entre dos señales para cada frecuencia del espectro. La coherencia proporciona valores entre 0 y 1 con una resolución frecuencial que puede variar en función de los parámetros del análisis. Un valor próximo a 1 indica una gran similitud, mientras que valores en torno a 0 indican ausencia de relación. A pesar de todo, los valores de coherencia dependen de la amplitud de la covarianza entre las señales, por lo que si se desea ignorar la relación de amplitudes y quedarse únicamente con la relación de fase entre dos señales (que representa de forma más limpia la sincronización), puede calcularse la coherencia de fase. Ambas técnicas se basan en el análisis de Fourier, y son medidas estáticas, aunque extensibles a versiones que logran describir la evolución temporal de las mismas. Si se desea obtener una medida dinámica, se puede calcular la eventrelated coherence (coherencia en relación con un evento $)^{18}$, que representa cómo varía la coherencia a lo largo del tiempo, o la coherencia basada en transformadas tiempo-frecuencia (como las wavelets), que proporciona un mapa en tiempo y frecuencia de la coherencia entre dos señales. 


\section{Herramientas de estudio de cambios oscilatorios en relación con un estímulo o evento}

Clásicamente, las respuestas neurofisiológicas a un estímulo o evento se han obtenido por promediación (potenciales evocados). La promediación únicamente deja visibles aquellos componentes de la respuesta que mantienen una relación temporal más o menos constante con respecto al estímulo o evento. Sobre esta señal promediada pueden aplicarse todas las herramientas descritas en apartados anteriores (transformadas de Fourier, transformadas tiempo-frecuencia, coherencia, coherencia de fase...). La relación constante de fase de una respuesta con el estímulo puede estudiarse asimismo mediante herramientas que la cuantifican en cada uno de los barridos individuales (la denominada intertrial coherence o phase-locking factor) ${ }^{19}$. Sin embargo, no todos los cambios que ocurren en relación con un estímulo o evento guardan la relación temporal constante necesaria para aparecer en la promediación. A estos cambios se les denomina "inducidos" por el estímulo o evento, en contraposición a los "evocados" que sí aparecen tras la premediación ${ }^{17}$. Un ejemplo fácil de entender lo constituye la atenuación del ritmo alfa occipital al abrir los ojos. Si promediáramos múltiples barridos utilizando el movimiento de cierre de ojos como evento, mediante promediación no obtendríamos ninguna señal relevante, ya que en el momento de la apertura de los ojos, la fase (el momento del ciclo) de la oscilación alfa será variable en cada barrido, y tenderá a atenuarse al promediar. Como alternativa, se puede determinar la amplitud de la señal en cada momento del barrido (sin tener en cuenta la fase) y promediar esas amplitudes. Existen distintos procedimientos que permiten detectar estos fenómenos de este tipo. La aproximación más simple consiste en rectificar la señal (habitualmente enventanándola para realizar un suavizado), con lo que se elimina parte de la fase (en esto se basan las técnicas denominadas ERD/ ERS -del inglés event-related desynchronization/synchronization ${ }^{20}$-. Otra solución más sofisticada emplearía transformadas tiempo-frecuencia sobre cada barrido individual, y posteriormente promediaría los mapas de energía que se obtienen de cada uno (prescindiendo de la información de fase $)^{21,22}$.

\section{OSCILACIONES ANORMALES EN LA ENFERMEDAD DE PARKINSON}

Las alteraciones en la actividad oscilatoria cerebral pueden jugar un papel fisiopatológico importante en varias enfermedades neurológicas y psiquiátricas, entre las que destaca la enfermedad de Parkinson (EP). La colocación de estimuladores cerebrales profundos en distintos núcleos de los ganglios basales para tratar los síntomas de diversas enfermedades ha permitido estudiar directamente en el hombre la actividad oscilatoria a nivel de estas estructuras y su relación con la actividad cortical, accesible desde la superficie de forma no invasiva. Estos registros han puesto de manifiesto la existencia de actividad oscilatoria anómala a distintas frecuencias, específica y relacionada con la fisiopatología de la enfermedad.

En pacientes con enfermedad de Parkinson, se observa en situación OFF (sin tratamiento) una intrusión de actividad oscilatoria anómala en los ganglios basales (núcleo subtalámico y parte interna del globo pálido) a frecuencias alrededor de $20 \mathrm{~Hz}$ que disminuye o desaparece al administrar fármacos dopaminérgicos ${ }^{23,24}$ (Fig. 3). Una actividad similar ha sido hallada en pacientes sin enfermedad de Parkinson tratados con tetrabenazina, fármaco que produce una deplección dopaminérgica ${ }^{25}$. En situación ON (tras administrar medicación) desaparece esta actividad beta y aparece una actividad oscilatoria de $60-80 \mathrm{~Hz}^{23,24}$. En menor medida existen también cambios de la actividad a frecuencias tan altas como $300 \mathrm{~Hz}^{26}$. Cuando se hacen estudios de coherencia se observa una sincronización a estas frecuencias (11-30 Hz en OFF y 60-80 en ON) entre el núcleo subtálamico y el globo pálido interno y la corteza motora ${ }^{27-29}$. 
Estudios con transformadas tiempofrecuencia han permitido demostrar que la actividad oscilatoria de $11-30 \mathrm{~Hz}$ se modifica durante la realización de movimientos balísticos siguiendo unos cambios bastante parejos a los observados en corteza cerebral ${ }^{30,31}$.

Desde hace más de 50 años se conoce que existen cambios en la actividad oscilatoria cortical en relación con el movimiento, en sujetos normales ${ }^{32}$. El ejemplo más evidente es la desaparición del ritmo mu al mover u observar un movimiento, pero a lo largo de las dos últimas décadas se han descrito muchos más cambios, abarcando un rango más amplio de frecuencias. La realización de un movimiento conlleva una marcada reducción de la actividad en el rango alfa y beta sobre áreas motoras, que comienza incluso más de un segundo antes del inicio del movimiento ${ }^{22,33}$.Tras el movimiento, se observa un "rebote» (un incremento que sigue al decaimiento observado durante la preparación y ejecución del movimiento) de la actividad beta ${ }^{34}$. Durante la ejecución propiamente dicha, se puede observar un incremento gamma sobre corteza motora primaria, evidente sobre todo en registros de electrocorticografía $a^{35}$. Aunque algunos cambios aparecen también durante movimientos pasivos (lo que sugiere una contribución de las aferencias sensitivas $)^{21,36}$, se admite que en los cambios beta existe un indudable componente motor ${ }^{37}$.

En el núcleo subtalámico de pacientes parkinsonianos se observa una desincronización de la actividad antes y durante la realización del movimiento que es seguida por un incremento al finalizar el movimiento. Esta disminución de la actividad beta se ve tanto en situación ON como $\mathrm{OFF}^{30,31,38}$. Pero al existir un gran aumento de beta anómalo en situación OFF, la disminución inducida por el acto motor no es suficiente para eliminarla. En situación ON se observa un incremento durante la ejecución del movimiento en la actividad de $60-80 \mathrm{~Hz}$. Igualmente existen cambios en otras situaciones como la observación del movimiento o durante el sueño ${ }^{39}$.
Las oscilaciones anómalas de los ganglios basales en la enfermedad de Parkinson no son hallazgos casuales o espurios sino que parecen tener un papel fisiopatológico ${ }^{40}$. La amplitud de la actividad beta anormal observada en el núcleo subtalámico de pacientes con enfermedad de Parkinson se correlaciona con la rigidez y la bradicinesia presentes en estos pacientes (aunque no con el temblor) ${ }^{41}$. La hipótesis de que la estimulación profunda provoca cambios en la actividad oscilatoria anómala de los ganglios basales de los pacientes con EP gana cada día más adeptos. De hecho, se ha descrito que la estimulación a $20 \mathrm{~Hz}$ en núcleo subtalámico provoca un aumento de la sincronización de la actividad en el pálido medial a dicha frecuencia sin mejora de la sintomatología. Sin embargo, estimulando a $70-80 \mathrm{~Hz}$ se produce una sincronización a esta frecuencia con una importante mejoría motora ${ }^{42,43}$. No es por tanto descabellado considerar que los cambios en la actividad oscilatoria y en la sincronización en los ganglios basales se acompañan de anomalías de la actividad oscilatoria en las cortezas que a su vez puede alterar el reclutamiento de unidades motoras en el músculo. Los mecanismos por los cuales surge esta actividad oscilatoria no son conocidos pero podrían estar relacionados con el hecho de que en la EP, los elementos que conforman la red constituida por los ganglios de la base y la corteza se ven modificadas. En este sentido, en la enfermedad de Parkinson existe una disminución de la inhibición en la corteza motora y a otros niveles del sistema nervioso. Si consideramos la dinámica cerebral dentro el marco de los sistemas complejos, se podría hipotetizar que estas modificaciones alejan al sistema de su punto de trabajo, por lo que los mecanismos de regulación y auto-organización dan lugar a una serie de modificaciones en la dinámica del sistema que hacen emerger las oscilaciones anómalas descritas y que sumergen al sistema en un estado de hiper/hiposincronización que lo saca de su equilibrio y por tanto dificulta su función (Fig. 4). 


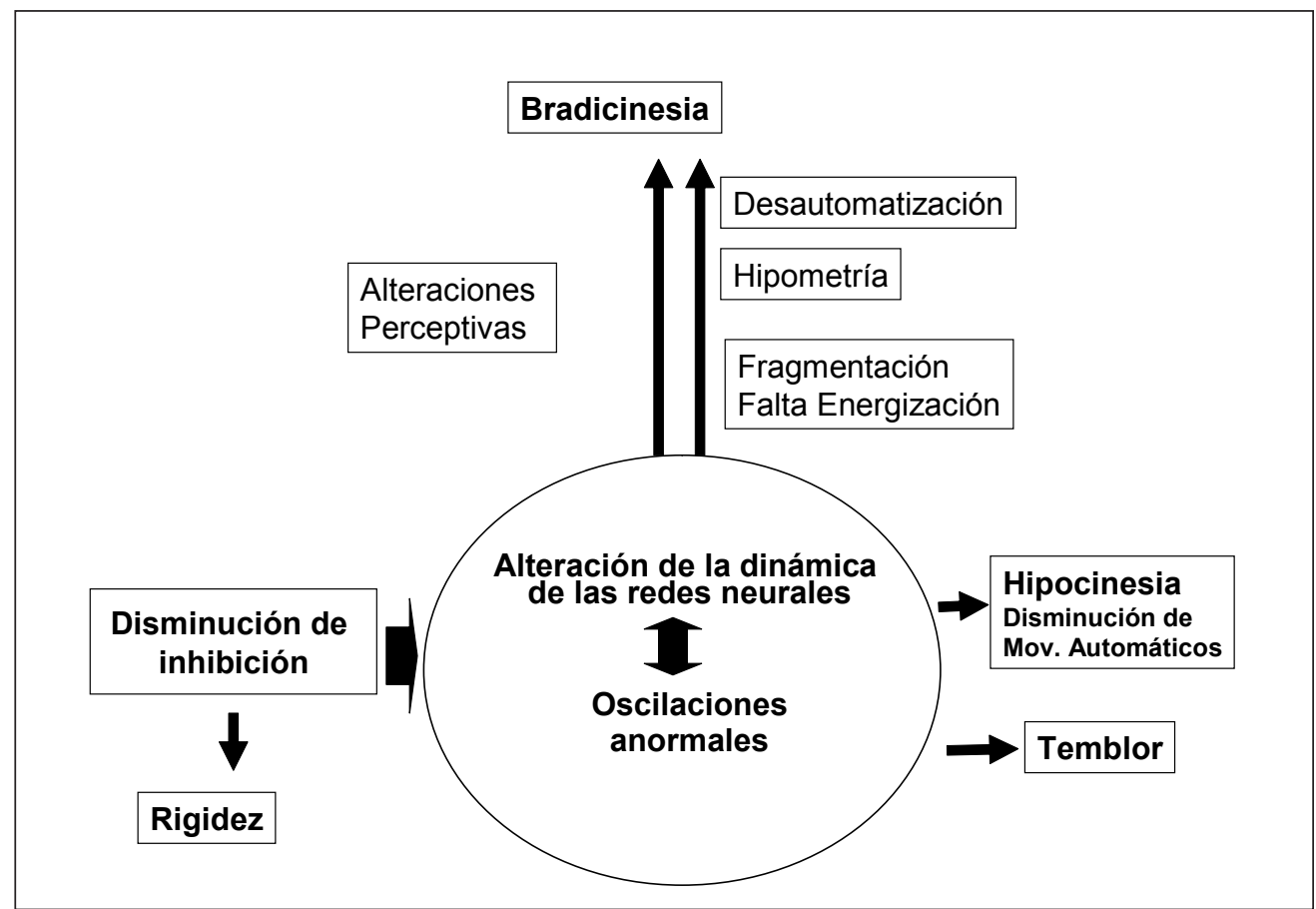

Figura 4. Diagrama esquematizando el papel fisiopatológico de las oscilaciones en la enfermedad de Parkinson.

A pesar de todo, la aparición de patrones oscilatorios anormales en los ganglios basales no es exclusiva de la enfermedad de Parkinson. En pacientes con distonía, se ha descrito la aparición de actividad theta excesiva en el globo pálido interno ${ }^{44}$. Es probable que en los próximos años, con la ampliación de las indicaciones de la estimulación cerebral profunda de los ganglios basales a otras patologías, se describan nuevos hallazgos de alteraciones específicas de la actividad oscilatoria en otros trastornos.

\section{OSCILACIONES Y EPILEPSIA}

Se acepta que la actividad epileptiforme se genera por una anormal y excesiva sincronización de las descargas de una red neuronal, que posteriormente se propaga a través de subredes conectadas entre sí. Las descargas síncronas generan una serie de activaciones patológicas que se mez- clan con oscilaciones fisiológicas. Esta actividad anormal es registrada por el EEG y la MEG en forma de puntas epileptiformes con actividad oscilatoria a frecuencias rápidas superpuesta en el periodo intercríti$\mathrm{co}^{45} \mathrm{y}$ en forma de actividad rítmica a diferentes frecuencias durante las crisis.

En pacientes con epilepsia las oscilaciones a frecuencia theta parecen tener especial relevancia. Por una parte se puede registrar actividad theta anormal en relación con esa tendencia a la hipersincronía cortical y, por otra parte, la ausencia de oscilaciones fisiológicas a frecuencias theta se ha relacionado con déficits que presentan estos pacientes. Así, durante la fase crítica uno de los patrones electroencefalográficos más frecuentes es la actividad theta rítmica ${ }^{46,47}$. Además, en pacientes con epilepsia generalizada se ha descrito que durante el periodo interictal existe un aumento difuso de la potencia y sincronía de las oscilaciones corticales a frecuencia the- 
ta. La sincronía se produce especialmente a nivel interhemisférico $\mathrm{y}$, aunque no ha podido ser demostrado por el momento, se hipotetiza que el tálamo juega un papel importante en dicha sincronización ${ }^{48}$. Por otra parte, mediante estudios experimentales se ha asociado la pérdida de oscilaciones theta normales en el hipocampo de pacientes con epilepsia medial temporal con el déficit de memoria que presentan estos pacientes, incluso en etapas presintomáticas ${ }^{49}$.

En los últimos años las oscilaciones a altas frecuencias, por encima de $80 \mathrm{~Hz}$, han adquirido especial relevancia en la epilepsia debido a su posible papel en la epileptogénesis. Las oscilaciones a $80-200 \mathrm{~Hz}$ denominadas "ripples» (R) y especialmente las oscilaciones entre 200 y $500 \mathrm{~Hz}$ denominadas "fast ripples» (FR) se han registrado mediante electrodos profundos, tanto en el periodo interictal ${ }^{50,51}$ como durante las cri$\mathrm{sis}^{52}$, en pacientes con epilepsia focal. Estas oscilaciones a frecuencias rápidas se han registrado en el foco epiléptico en diversos tipos de epilepsias ${ }^{53} y$ con frecuencia aparecen de forma independiente de las puntas epilépticas observadas en el EEG ${ }^{54}$. Estas oscilaciones aumentan progresivamente en potencia antes de las crisis ${ }^{55}$ a diferencia de las puntas no aumentan en frecuencia después de las crisis pero sí lo hacen al disminuir la medicación antiepiléptica ${ }^{56}$. Todos estos hallazgos hacen suponer que las oscilaciones a altas frecuencias son un marcador de epileptogenicidad mejor que las puntas. De hecho, se ha descrito que la inhibición de frecuencias muy altas $(>200$ $\mathrm{Hz}$ ) después de las puntas intercríticas es un fenómeno mucho más limitado en el espacio que la punta en $s^{5}{ }^{57}$.

\section{SINCRONIZACIÓN DE FASE Y OSCILACIONES EN LA ESQUIZOFRENIA}

Algunas teorías actuales sobre la esquizofrenia mantienen que varios de los hallazgos claves en esta enfermedad podrían deberse a un síndrome de desconexión tanto dentro de cada una de las diversas áreas corticales como en las conexiones entre ellas $^{58}$. Las alteraciones en la actividad oscilatoria cortical podrían representar el correlato funcional de esta desconexión en redes corticales, y por tanto, sustentar la fragmentación de mente y comportamiento característica de la esquizofrenia ${ }^{59}$. Por otra parte, la desconexión funcional entre distintas redes corticales podría reflejar alteraciones en la conectividad anatómica, como sugieren las anomalías de sustancia blanca encontradas en esta enfermedad ${ }^{60}$.

Se han descrito múltiples alteraciones en la actividad oscilatoria en la esquizofrenia, abarcando bandas de frecuencia muy amplias, desde theta hasta gamma. En general, se observa en la mayoría de los trabajos una tendencia hacia una menor capacidad de sincronización que concuerda con la hipótesis anterior. En la banda theta, las alteraciones observadas en la sincronización han sido relacionadas con las funciones, que las oscilaciones en esta banda de frecuencias parecen tener en la memoria de trabajo (working memory) ${ }^{61}$. Asimismo, se ha propuesto que la falta de capacidad para generar sincronización de larga distancia entre el lóbulo frontal y el temporal podría estar relacionada con la presencia de alucinaciones auditivas en estos pacientes, debido a un menor control frontal de la actividad temporal ${ }^{62}$. Aunque también se han observado alteraciones en la sincronización beta durante tareas perceptivas $^{63}$, los cambios más estudiados son los relacionados con la banda gamma. Estos cambios incluyen una reducción de la energía y de la sincronización en esta banda, en comparación con los controles, durante tareas perceptivas y/o cognitivas ${ }^{64-66}$. Dentro de las distintas modalidades de respuesta estudiada, destacan por su facilidad de aplicación y la consistencia de los hallazgos los potenciales evocados auditivos de estado estable. Se ha descrito tanto una reducción de amplitud como de sincronización de fase en las respuestas a una frecuencia de estimulación de $40 \mathrm{~Hz}^{67}$; aunque se acepta en general que esta disminución es independiente del tratamiento, un trabajo reciente sugiere que las respuestas pueden verse alteradas por algunos neurolép- 
ticos ${ }^{68}$. La disminución de actividad gamma en esta enfermedad no afecta únicamente a respuestas "evocadas" (ligadas en fase) como los potenciales de estado estable, sino también a la actividad "inducida" (independiente de la fase) que se observa tras estímulos auditivos en paradigmas go/no go (de tipo "oddball» o P300) ${ }^{69}$.

Se ha comentado en apartados anteriores cómo la sincronización gamma puede estar implicada en fenómenos de integración cortical a distintos niveles. La dificultad para lograr una sincronización adecuada de las distintas estructuras neurales que oscilan a estas frecuencias podría, por tanto, jugar un papel clave en la fisiopatología de la esquizofrenia.

\section{CONSIDERACIONES FINALES}

La actividad oscilatoria cortical o de otras estructuras se encuentra alterada en distintas patologías. Estas oscilaciones anormales pueden ser el resultado de una dinámica alterada de la red neural y juegan un papel fisiopatológico en los síntomas de estas entidades. En un futuro, el adecuado estudio neurofisiológico de las oscilaciones ya sean basales, inducidas por estímulos o como consecuencia de un proceso perceptivo, cognitivo o motor, posiblemente pueda tener un papel diagnóstico. Igualmente es posible hipotetizar que su modificación por estimulación eléctrica o magnética u otros agentes podría ser una herramienta terapéutica de utilidad como hoy en día ya lo es la estimulación del núcleo subtalámico en la enfermedad de Parkinson y otros movimientos anormales

\section{Agradecimientos}

Trabajo parcialmente financiado por UTE proyecto CIMA y Fondo de Investigaciones Sanitarias (FIS 070034).

\section{BIBLIOGRAFÍA}

1. Gray CM, Konig P, Engel AK, Singer W. Oscillatory responses in cat visual cortex exhibit inter-columnar synchronization which reflects global stimulus properties. Nature 1989; 338 : 334-337.

2. SINGER W. Synchronization of cortical activity and its putative role in information processing and learning. Annu Rev Physiol 1993; 55: 349-374.

3. BARLOW HB. Single units and sensation: a neuron doctrine for perceptual psychology? Perception 1972; 1: 371-394.

4. PALm G. Neural assembles. Springer-Verlag, 1982.

5. Von der Malsburg C, Schneider W. A neural cocktail-party processor. Biol Cybern 1986; 54: 19-40.

6. VON DER MALSBURG C. Binding in models of perception and brain function. Curr Opin Neurobiol 1995; 5: 520-526.

7. Jefferys JG, Traub RD, Whittington MA. Neuronal networks for induced ' $40 \mathrm{~Hz}$ ' rhythms. Trends Neurosci 1996; 19: 202-208.

8. Buzsaki G, ChrobaK JJ. Synaptic plasticity and self-organization in the hippocampus. Nat Neurosci 2005; 8: 1418-1420.

9. SteRIADE M. Impact of network activities on neuronal properties in corticothalamic systems. J Neurophysiol 2001; 86: 1-39.

10. Csicsvari J, Jamieson B, Wise KD, Buzsaki G. Mechanisms of gamma oscillations in the hippocampus of the behaving rat. Neuron 2003; 37: 311-322.

11. Chialvo DR. Critical brain networks. Physica A 2009; 340: 756 .

12. BAK P. How nature works: The science of selforganised criticality. New York: Copernicus Press, 1996.

13. Bak P, Tang C, Wiesenfeld K. Self-organized criticality: An explanation of the $1 / \mathrm{f}$ noise. Phys Rev Lett 1987; 59: 381-384.

14. Linkenkaer-Hansen K, Nikouline VV, Palva JM, ILMONIEMI RJ. Long-range temporal correlations and scaling behavior in human brain oscillations. J Neurosci 2001; 21: 1370-1377.

15. Castellanos NP, Malmierca E, Nuñez A, Makarov VA. Corticofugal modulation of the tactile response coherence of projecting neurons in the gracilis nucleus. J Neurophysiol 2007; 98 : $2537-2549$

16. Cohen L. Time-frequency distributions. A review. Proc IEEE 1989; 77: 941-981.

17. Gurtubay IG, Alegre M, Labarga A, Malanda A, IrIarte J, ARTiedA J. Gamma band activity in an auditory oddball paradigm studied with the Wavelet Transform. Clin Neurophysiol 2001; 112: 1219-1228. 
18. Andrew C, Pfurtscheller G. Event-related coherence as a tool for studying dynamic interaction of brain regions. Electroencephalogr Clin Neurophysiol 1996; 98: 144-148.

19. Tallon-Baudry C, Bertrand O, Delpuech C, PerNIER J. Stimulus specificity of phase-locked and non-phase-locked $40 \mathrm{~Hz}$ visual responses in human. J Neurosci 1996; 16: 4240-4249.

20. Pfurtscheller G, Aranibar A. Event-related cortical desynchronization detected by power measurements of scalp EEG. Electroencephalogr Clin Neurophysiol 1977; 42: 817826.

21. Alegre M, Labarga A, Gurtubay IG, Iriarte J, Malanda A, Artieda J. Beta EEG changes during passive movements: sensory afferences contribute to the beta event-related desynchronization in humans. Neurosci Lett 2002; 331: 29-32.

22. Alegre M, Labarga A, Gurtubay IG, Iriarte J, Malanda A, Artieda J. Movement-related changes in cortical oscillatory activity in ballistic, sustained and negative movements. Exp Brain Res 2003; 148: 17-25.

23. Alonso-Frech F, Zamarbide I, Alegre M, RodrIguez-Oroz MC, Guridi J, Manrique M et al. Slow oscillatory activity and levodopa-induced dyskinesias in Parkinson's disease. Brain 2006; 129: 1748-1757.

24. Priori A, Foffani G, Pesenti A, Tamma F, Bianchi AM, Pellegrini M et al. Rhythm-specific pharmacological modulation of subthalamic activity in Parkinson's disease. Exp Neurol 2004; 189: 369-379.

25. Kuhn AA, Brucke C, Schneider GH, Trottenberg T, KIVI A, KuPSCH A et al. Increased beta activity in dystonia patients after drug-induced dopamine deficiency. Exp Neurol 2008; 214: 140-143.

26. Foffani G, Priori A, Egidi M, Rampini P, Tamma F, CAPUTO E et al. $300-\mathrm{Hz}$ subthalamic oscillations in Parkinson's disease. Brain 2003; 126 : 2153-2163.

27. Williams D, Tujssen M, Van Bruggen G, Bosch A, Insola A, Di Lazzaro V et al. Dopamine-dependent changes in the functional connectivity between basal ganglia and cerebral cortex in humans. Brain 2002; 125: 1558-1569.

28. Trottenberg T, Fogelson N, Kuhn AA, Kivi A, Kupsch A, SchneIDER GH et al. Subthalamic gamma activity in patients with Parkinson's disease. Exp Neurol 2006; 200: 56-65.

29. Lalo E, Thobois S, Sharott A, Polo G, Mertens $\mathrm{P}$, Pogosyan A et al. Patterns of bidirectional communication between cortex and basal ganglia during movement in patients with Parkinson disease. J Neurosci 2008; 28: 30083016.

30. Alegre M, Alonso-Frech F, Rodríguez-Oroz MC, Guridi J, Zamarbide I, Valencia M et al. Movement-related changes in oscillatory activity in the human subthalamic nucleus: ipsilateral vs. contralateral movements. Eur J Neurosci 2005 ; 22 : 2315-2324.

31. Devos D, Szurhaj W, Reyns N, Labyt E, Houdayer E, BourriEz JL et al. Predominance of the contralateral movement-related activity in the subthalamo-cortical loop. Clin Neurophysiol 2006; 117: 2315-2327.

32. Gastaut H. Etude électrocorticographique de la réactivité des rythmes rolandiques. Rev Neurol Paris 1952; 87: 176-182.

33. Pfurtscheller G, Aranibar A. Evaluation of event-related desynchronization (ERD) preceding and following voluntary self-paced movement. Electroencephalogr Clin Neurophysiol 1979; 46: 138-146.

34. Pfurtscheller G. Event-related synchronization (ERS): an electrophysiological correlate of cortical areas at rest. Electroencephalogr Clin Neurophysiol 1992; 83: 62-69.

35. Crone NE, Miglioretti DL, Gordon B, Lesser RP. Functional mapping of human sensorimotor cortex with electrocorticographic spectral analysis. II. Event-related synchronization in the gamma band. Brain 1998; 121: 2301-2315.

36. Cassim F, Monaca C, Szurhaj W, Bourriez JL, DeFebvre L, Derambure $P$ et al. Does post-movement beta synchronization reflect an idling motor cortex? Neuroreport 2001; 12: 38593863.

37. Alegre M, Álvarez-Gerriko I, Valencia M, Iriarte J, ARTiEdA J. Oscillatory changes related to the forced termination of a movement. Clin Neurophysiol 2008; 119: 290-300.

38. Androulidakis AG, Brucke C, Kempf F, Kupsch A, AzIz T, Ashran K et al. Amplitude modulation of oscillatory activity in the subthalamic nucleus during movement. Eur J Neurosci 2008; 27: $1277-1284$.

39. Urrestarazu E, Iriarte J, Alegre M, Clavero P, Rodríguez-Oroz MC, Guridi J et al. Beta activity in the subthalamic nucleus during sleep in patients with Parkinson's disease. Mov Disord 2008; 24: 254-260.

40. Brown P. Oscillatory nature of human basal ganglia activity: relationship to the pathophysiology of Parkinson's disease. Mov Disord 2003; 18: 357-363. 
41. Kuhn AA, Tsui A, Aziz T, Ray N, Brucke C, KuPSCH A et al. Pathological synchronisation in the subthalamic nucleus of patients with Parkinson's disease relates to both bradykinesia and rigidity. Exp Neurol 2009; 215: 380387.

42. Brown P, Mazzone P, Oliviero A, Altibrandi MG, Pilato F, Tonali PA et al. Effects of stimulation of the subthalamic area on oscillatory pallidal activity in Parkinson's disease. Exp Neurol 2004; 188: 480-490.

43. Chen CC, Litvak V, Gilbertson T, Kuhn A, Lu CS, LEE ST et al. Excessive synchronization of basal ganglia neurons at $20 \mathrm{~Hz}$ slows movement in Parkinson's disease. Exp Neurol 2007; 205 : 214-221.

44. Silberstein P, Kunn AA, Kupsch A, Trottenberg T, Krauss JK, Wohrle JC et al. Patterning of globus pallidus local field potentials differs between Parkinson's disease and dystonia. Brain 2003; 126: 2597-2608.

45. Guggisberg AG, Kirsch HE, Mantle MM, Barbaro NM, NAGARAJAN SS. Fast oscillations associated with interictal spikes localize the epileptogenic zone in patients with partial epilepsy. Neuroimage 2008; 39: 661-668.

46. Li X, Jefrerys JG, Fox J, Yao X. Neuronal population oscillations of rat hippocampus during epileptic seizures. Neural Netw 2008; 21 : 1105-1111.

47. Dericioglu N, SAYgi S. Ictal scalp EEG findings in patients with mesial temporal lobe epilepsy. Clin EEG Neurosci 2008; 39: 20-27.

48. Clemens B. Pathological theta oscillations in idiopathic generalised epilepsy. Clin Neurophysiol 2004; 115: 1436-1441.

49. Chauviere L, Rafrafi N, Thinus-Blanc C, BartoLOMEI F, Esclapez M, Bernard C. Early deficits in spatial memory and theta rhythm in experimental temporal lobe epilepsy. J Neurosci 2009; 29: 5402-5410.

50. Bragin A, Wilson CL, Staba RJ, Reddick M, Fried I, ENGEL J, JR. Interictal high-frequency oscillations $(80-500 \mathrm{~Hz})$ in the human epileptic brain: entorhinal cortex. Ann Neurol 2002; 52: 407-415.

51. Urrestarazu E, Chander R, Dubeau F, Gotman J. Interictal high-frequency oscillations (100$500 \mathrm{~Hz}$ ) in the intracerebral EEG of epileptic patients. Brain 2007; 130: 2354-2366.

52. Jirsch JD, Urrestarazu E, Levan P, Olivier A, Dubeau F, Gotman J. High-frequency oscillations during human focal seizures. Brain 2006; 129: 1593-1608.
53. Jacobs J, Levan P, Chatillon CE, Olivier A, Dubeau F, Gotman J. High frequency oscillations in intracranial EEGs mark epileptogenicity rather than lesion type. Brain 2009; 132: 1022-1037.

54. Jacobs J, Levan P, Chander R, Hall J, Dubeau F, Gotman J. Interictal high-frequency oscillations $(80-500 \mathrm{~Hz})$ are an indicator of seizure onset areas independent of spikes in the human epileptic brain. Epilepsia 2008; 49: 18931907.

55. Khosravani H, Mehrotra N, Rigby M, Hader WJ, PinNegar CR, Pillay N et al. Spatial localization and time-dependant changes of electrographic high frequency oscillations in human temporal lobe epilepsy. Epilepsia 2009; 50: 605-616.

56. Zijlmans M, Jacobs J, Zelmann R, Dubeau F, GotMAN J. High frequency oscillations and seizure frequency in patients with focal epilepsy. Epilepsy Res 2009 (en prensa); doi:10.1016/j. eplepsyres.2009.03.026.

57. Urrestarazu E, Jirsch JD, Levan P, Hall J, Avoli $\mathrm{M}$, Dubeau $\mathrm{F}$ et al. High-frequency intracerebral EEG activity (100-500 Hz) following interictal spikes. Epilepsia 2006; 47: 1465-1476.

58. UhlhaAs PJ, Singer W. Neural synchrony in brain disorders: relevance for cognitive dysfunctions and pathophysiology. Neuron 2006; 52: 155-168.

59. Uhlhaas PJ, Haenschel C, Nikolic D, Singer W. The role of oscillations and synchrony in cortical networks and their putative relevance for the pathophysiology of schizophrenia. Schizophr Bull 2008; 34: 927-943.

60. Kubicki M, McCarley R, Westin CF, Park HJ, MAIER S, KIKINIS R et al. A review of diffusion tensor imaging studies in schizophrenia. J Psychiatr Res 2007; 41: 15-30.

61. Schmiedt C, Brand A, Hildebrandt H, Basar-EroGLU C. Event-related theta oscillations during working memory tasks in patients with schizophrenia and healthy controls. Brain Res Cogn Brain Res 2005; 25: 936-947.

62. Ford JM, Mathalon DH. Corollary discharge dysfunction in schizophrenia: can it explain auditory hallucinations? Int J Psychophysiol 2005; 58: 179-189.

63. UhlhaAs PJ, Linden DE, Singer W, Haenschel C, LindNER M, MAURER K et al. Dysfunctional longrange coordination of neural activity during Gestalt perception in schizophrenia. J Neurosci 2006; 26: 8168-8175.

64. Spencer KM, Nestor PG, Niznikiewicz MA, SalisBury DF, Shenton ME, McCarley RW. Abnormal neural synchrony in schizophrenia. J Neurosci 2003; 23: 7407-7411. 
65. GonzÁlez-Hernández JA, Cedeno I, Pita-Alcorta C, Galán L, Aubert E, Figueredo-Rodríguez P. Induced oscillations and the distributed cortical sources during the Wisconsin card sorting test performance in schizophrenic patients: new clues to neural connectivity. Int J Psychophysiol 2003; 48: 11-24.

66. Kissler J, Muller MM, Fehr T, Rockstroh B, ELBERT T. MEG gamma band activity in schizophrenia patients and healthy subjects in a mental arithmetic task and at rest. Clin Neurophysiol 2000; 111: 2079-2087.

67. Light GA, Hsu JL, Hsieh MH, Meyer-Gomes K, Sprock J, Swerdlow NR et al. Gamma band oscillations reveal neural network cortical coherence dysfunction in schizophrenia patients. Biol Psychiatry 2006; 60: 12311240.

68. Mayner G, Ortuño F, López P, Alegre M, Valencia M, ARTIEDA J. Atypical neuroleptics may modify cortical oscillatory activity in schizophrenia. European Neuropsychopharmacology 2008; 18 (Suppl 4): S459-S460.

69. Haig AR, Gordon E, De Pascalis V, Meares RA, BAHRAMALI H, HaRris A. Gamma activity in schizophrenia: evidence of impaired network binding? Clin Neurophysiol 2000; 111: 14611468 . 